\title{
Origin of Irregularities in RR Lyrae Pulsations
}

\author{
Merieme Chadid \\ European Southern Observatory, Alonso de Cordova, 3107, P.O. 19001, \\ Santiago, Chile
}

\begin{abstract}
For the first time important irregularities in atmospheric pulsations of RR Lyrae itself are clearly detected. They occur from one pulsation cycle to another. Their origins cannot be directly explained by the Blazhko effect. We suggest that these irregularities are mostly provoked by strong shock waves propagating within the atmosphere.
\end{abstract}

\section{Introduction}

Up to now, atmospheric pulsating models of RR Lyrae stars assume that the motion of layers where the metallic and hydrogen lines are formed are strictly periodic. An appreciable fraction of RR Lyrae stars show noticeable variations in their luminosity and radial velocity curves over a period of about 100 pulsation cycles (Blazhko effect). Moreover, Chadid \& Gillet (1997) have confirmed a variation of the intensity of the hydrogen line emission over the Blazhko period of RR Lyrae itself. This variation is directly related to the shock wave intensity. Is it possible that shock waves induce some irregularities in the dynamical motion of the atmospheric layers?

\section{Detection and Origin of the RR Lyrae Irregularities}

A set of high spectral resolution observations of a Fe II line was obtained with the ELODIE spectrograph at the 1.93-m telescope at the Observatoire de HauteProvence, with a resolving power around 42000 , a time resolution between 0.9 and $1.2 \%$ of the pulsation period $(13 \mathrm{hr} 36 \mathrm{~min})$ and a signal-to-noise ratio around 50 . Fig. 1 shows a superposition of the full width at half maximum (FWHM) curves of the Fe II line at different Blazhko phases. To cover a complete pulsation period, 3 consecutive nights were required. From one night to another, a dispersion clearly appears when the FWHM is observed over two nights. We expect that it is not only due to the presence of the Blazhko effect, because in this case the estimated total amplitude over the Blazhko period ( $40.8 \mathrm{~d}$ ) would be much too large. This dispersion effect was observed at five different Blazhko phases. What is the physical effect responsible for this irregularity? From these observations (see Chadid 1999), we know that the radial velocity maximum is not the same for all the Blazhko phases. It occurs during the bump observed in the luminosity curve around the pulsation phase 0.70 , just before the minimum photospheric radius. This is the consequence of the progressive breaking of the infalling motion of the high atmosphere during the contraction. Fokin \& 


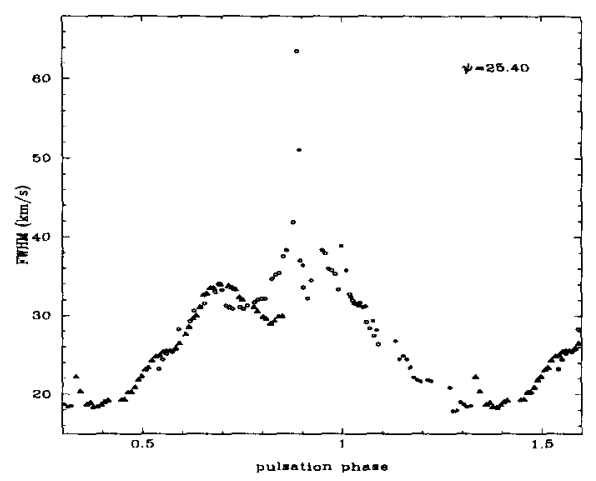

Figure 1. Full width half maximum (FWHM) obtained during 3 nights (black point: 1997 August 5; white circles: 1997 August 6; triangles: 1997 August 7) for the line Fe II $4923.921 \AA . \Psi=25.40$ is the Blazhko phase.

Gillet (1997) showed that a shock wave produces at this time an additional local compression of the atmospheric layers in which metallic lines are formed. This dynamical phenomenon induces a secondary photospheric acceleration which is certainly at the origin of the main irregularities detected into the FWHM curves. Indeed, because the FWHM changes of the Fe II line profile are very sensitive to physical conditions within the atmosphere (Chadid \& Gillet 1996), we must expect that FWHM irregularities are due to this dynamical atmospheric process.

\section{Conclusions and Future Work}

Some important irregularities occurring during successive pulsation cycles have been clearly indicated. They cannot be directly explained by the Blazhko effect which should only induce a more or less regular modification of radial velocity and FWHM curves versus the Blazhko period. Because there are two strong outward shock waves together with an infalling shock per pulsation period, we suggest that the observed irregularities must be mainly due to a nonlinear dynamical process between the motion of the highest and deepest atmospheric layers which are out of synchronization. Finally, our observations show that new detailed nonlinear nonadiabatic pulsation models of the atmospheres of RR Lyrae stars are now required to confirm the origin of these irregularities.

\section{References}

Chadid, M. 1999, A\&A, submitted

Chadid, M. \& Gillet, D. 1996, A\&A, 315, 475

Chadid, M. \& Gillet, D. 1997, A\&A, 319, 154

Fokin, A. B. \& Gillet, D. 1997, A\&A, 325, 1013 